\title{
Stack-Run Adaptive Wavelet Image Coding
}

\author{
A. Majid Awan ${ }^{1} \quad$ Nasir M. Rajpoot ${ }^{2} \quad$ S. Afaq Husain ${ }^{1}$ \\ ${ }^{1}$ Faculty of CSE, GIK Institute of Engg. S\&T, Pakistan \\ ${ }^{2}$ Dept. of Computer Science, University of Warwick, UK \\ email: gcs0105@giki.edu.pk, nasir@dcs.warwick.ac.uk, syed_a_h@giki.edu.pk
}

Wavelets are not well-suited to represent oscillatory patterns, a special form of texture. However, results from various image coding methods based on wavelet packets, such as [1], show that they are particularly good in coding images with oscillatory patterns. In this paper, we present an adaptive wavelet transform based image coder which employs a stack-run representation for quantized transform coefficients in order to benefit from the intra-subband redundancies. Our compression algorithm can be divided into four parts: In the first part, an adaptive wavelet packet basis is selected for representing the given image using certain entropy-based cost function. Next, the wavelet packet coefficients are quantized using an optimal scalar quantizer for Laplacian distribution. In the third part, we represent the quantized coefficients with stack-run coding [3] generating a redundant symbol stream. Finally, this symbol stream is entropy coded using a higher order arithmetic coder. The coder variations were based on three different filters: symmlet-8, biorthogonal $17 / 11$, and biorthogonal 9/7 filters. Each of these variations employed three additive cost functions for basis selection: Coifman-Wickerhauser (CW) entropy, energy, and $l_{1}$-norm. Coding gains achieved by our SRWP coder over both SPIHT [2] and FAWP [1] coders are noticeable, especially for Barbara (1.2dB over SPIHT and $0.4 \mathrm{~dB}$ over FAWP). Another interesting result is the consistently better performance of the symmlet- 8 filters over filters in popular use.

\section{References}

[1] F.G. Meyer, A.Z. Averbuch, and J-O. Strömberg. Fast adaptive wavelet packet image compression. IEEE Transactions on Image Processing, 9(5):792-800, May 2000 .

[2] A. Said and W.A. Pearlman. A new fast and efficient image codec based on set partitioning in hierarchical trees. IEEE Transactions on Circuits \& Systems for Video Technology, 6:243-250, June 1996.

[3] M.J. Tsai, J.D. Villasenor, and F. Chen. Stack-run image coding. IEEE Transactions on Circuits and Systems for Video Technology, 6:519-521, October 1996. 